\title{
In Situ Heating to Investigate Phase Transformations in Individual Powder Particles of a Gas-Atomized Icosahedral-Phase-Strengthened Al Alloy
}

\author{
H.R. Leonard ${ }^{1 *}$, S. Rommel ${ }^{1}$, S. Vijayan ${ }^{1}$, T.J. Watson ${ }^{2}$, T. Policandriotes ${ }^{3}$, and M. Aindow ${ }^{1}$ \\ 1. Department of Materials Science and Engineering, Institute of Materials Science, University of \\ Connecticut, Storrs, CT 06269, USA. \\ 2. Pratt \& Whitney, Materials \& Process Engineering, East Hartford, CT 06108, USA. \\ 3. Collins Aerospace, Windsor Locks, CT 06096, USA. \\ * Corresponding author: Hannah.Leonard@UConn.edu
}

Gas atomization is a common method of producing metallic alloy powders by using a high-pressure gas to break up a stream of molten alloy. A fine dispersion of spherical powder particles with a constant average composition can be produced even from complex multi-component alloys. The other notable aspect of gas atomization is the high cooling rate $\left(10^{4}-10^{8} \mathrm{~K} / \mathrm{s}\right)$, which results in the formation of metastable phases and non-equilibrium microstructures in the powder particles. It is of interest to study how these radically different phases and microstructures evolve under thermal processing through in situ transmission election microscopy (TEM). However, powder particles larger than $\approx 5 \mu \mathrm{m}$ are particularly challenging samples to study by in situ TEM.

A recently-developed approach was used to prepare cross sections through individual powder particles for in situ heating experiments [1]. This approach uses focused ion beam (FIB) milling to prepare a sitespecific sample and to transfer it to a micro-electromechanical systems (MEMS) based heating holder (Figure 1). The MEMS heating holder consists of a heat spreader plate with an array of $5 \mu \mathrm{m}$ windows, and the electron transparent region of the sample is placed over one of these windows. In this manner we can observe the dynamic processes that occur as the non-equilibrium phases and microstructures evolve via a variety of thermally activated mechanisms.

For the experiment shown here, an Al-Cr-Mn-Co-Zr alloy was selected. The gas atomized powder of this alloy exhibits a nano-composite FCC Al plus I-phase microstructure [2-4]. The I-phase is quasicrystalline and exhibits rotational symmetry but lacks translational symmetry. This unusual structure gives the Iphase a remarkable combination of properties including high strength, hardness, elastic modulus, as well as wear and corrosion resistance. The formation of quasicrystals requires a high cooling rate $\left(\sim 10^{-6} \mathrm{~K} / \mathrm{s}\right)$, which has limited their production, but gas atomization has shown promise for scale-up, and has promoted the development of their use in high-strength and corrosion-resistant aluminum alloys. Quasicrystals are inherently metastable, and in order to understand their thermal stability in the Al-Cr-Mn-Co-Zr alloy, a series of in situ studies has been conducted. In the example shown (Figure 2) the cross-sectional sample was heated to $500{ }^{\circ} \mathrm{C}$ and held for 31 minutes. EDXS revealed that the I-phase decomposed into an Al$\mathrm{Cr}-\mathrm{Mn}$ and an Al-Co phase. These in-situ data show that the decomposition of the I-phase appears to be associated with the ejection of Co.

References:

[1] S. Vijayan et al., Microsc Microanal 23 (2017), p. 708-716

[2] T.J Watson et al., Scripta Mater 123 (2016), p. 51-54.

[3] T.J Watson et al., Corros Sci 121 (2017), p. 133-138. 
[4] T.J Watson et al., Surf Coat Tech 324 (2017), p. 57-63.

[5] These studies were performed in the UConn/ Thermo Fisher Scientific Center for Advanced Microscopy and Materials Analysis (CAMMA).

a)

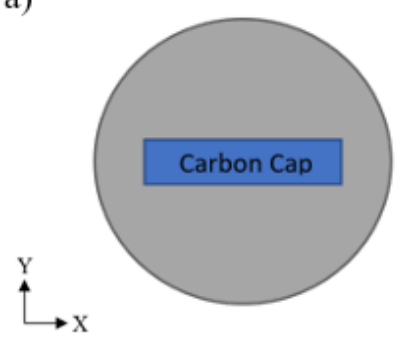

d)

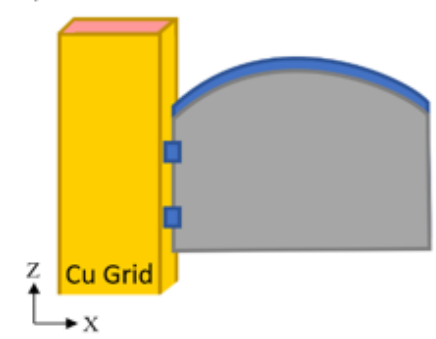

g) b)

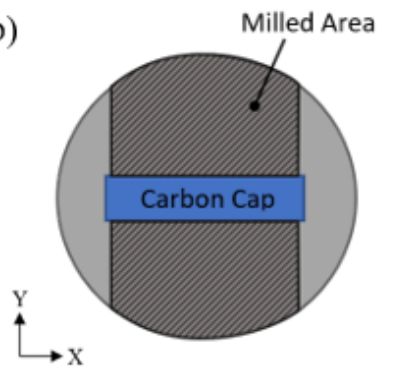

e)

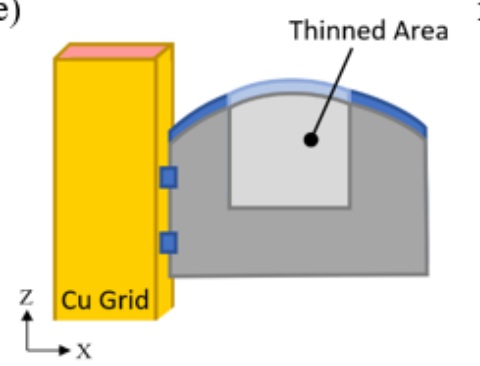

c)

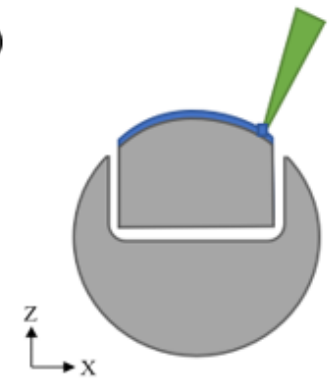

f)

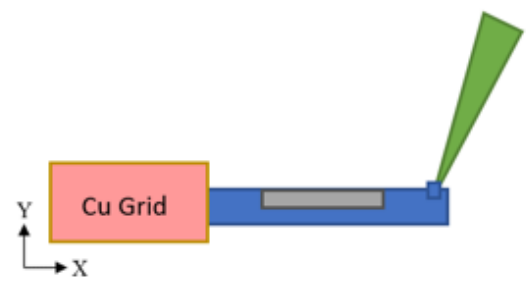

h)
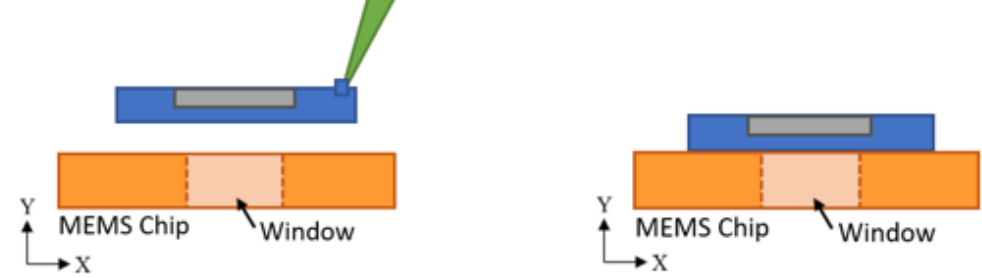

Figure 1. Schematic diagrams showing the steps in the FIB sample preparation technique: (a) carbon cap over the region of interest, (b) milled out lamella, (c) lift out, (d) attached to $\mathrm{Cu}$ grid, (e) final thinning, (f) detachment from grid, (g) placement on MEMS chip, and (h) the final configuration.
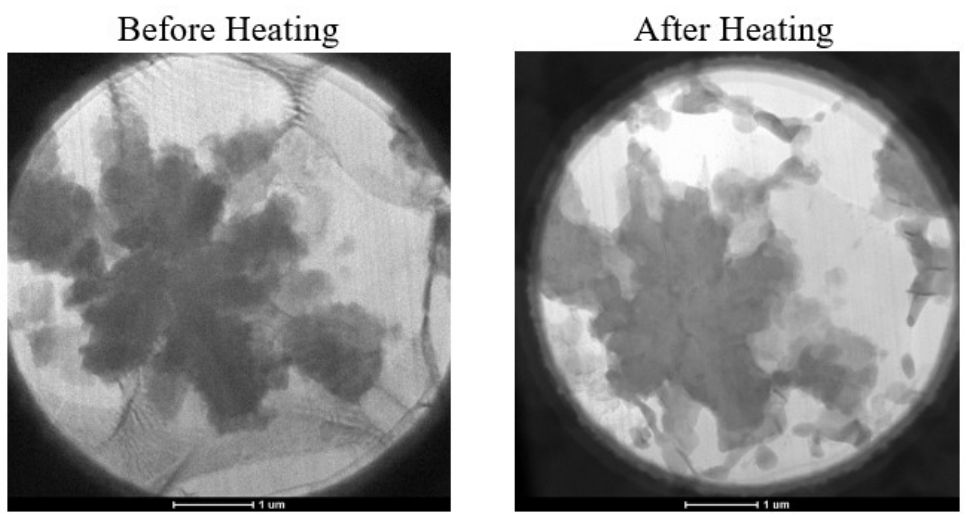

Figure 2. BF TEM images obtained from the region of interest before and after heating the specimen to $500{ }^{\circ} \mathrm{C}$ for half an hour. 